\title{
COMPARAÇÃO DE TÉCNICAS DE CONTROLE APLICADAS A UM SISTEMA DE LEVITAÇÃO MAGNÉTICA
}

\author{
Caio Igor Goncalves Chinelato
}

Universidade Federal do ABC - UFABC

Av. dos Estados, 5001, Bangu, 09210-971, Santo André, SP, Brasil

E-mail: caio_i_c@hotmail.com

Resumo: Neste trabalho é apresentado o estudo e implementação de três técnicas de controle aplicadas a um sistema de levitação magnética. Este é um sistema dinâmico não-linear e não pode ser controlado globalmente por técnicas de controle lineares tradicionais. As técnicas de controle usadas são PD (Proporcional-Derivativo) com o sistema linearizado no ponto de equilíbrio, linearização por realimentação e SDRE (State-Dependent Riccati Equation). $O$ objetivo deste trabalho é comparar o comportamento do sistema às diferentes técnicas de controle aplicadas. Resultados simulados com o software Matlab mostram a aplicação das técnicas de controle ao sistema.

Palavras-chave: Sistema de Levitação Magnética, Sistemas Dinâmicos Não-lineares, Controle $P D$, Linearização por realimentação, Controle SDRE.

\section{Introdução}

O sistema analisado é o sistema de levitação magnética [4]. O objetivo é manter suspensa no ar uma bola metálica através da variação do campo magnético. A corrente eletromagnética deve aumentar até a força magnética ser igual a força gravitacional atuando na bola. O sistema é mostrado na Figura 1 [6].

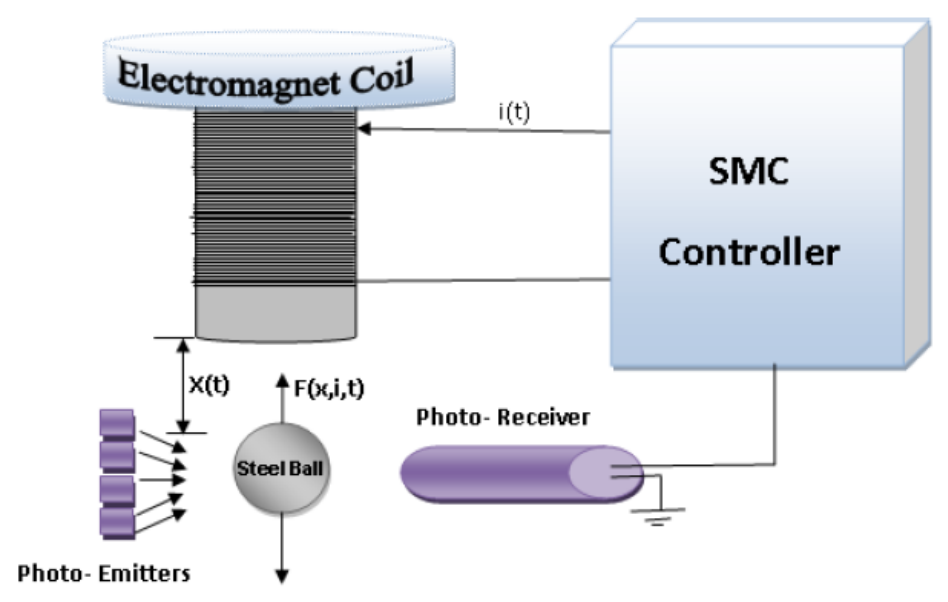

Figura 1: Sistema de levitação magnética [6].

A modelagem do sistema pode ser vista detalhadamente em [6]. O sistema no espaço de estados é dado por:

$$
\begin{aligned}
& \dot{X}_{1}=X_{2}=f_{1} \\
& \dot{X}_{2}=g-\frac{K}{m} \cdot \frac{X_{3}^{2}}{X_{1}^{2}}=f_{2} \\
& \dot{X}_{3}=\frac{-R \cdot X_{3}}{L_{1}+\frac{2 K}{X_{1}}}+\frac{2 K}{L_{1}+\frac{2 K}{X_{1}}} \cdot \frac{X_{2} X_{3}}{X_{1}^{2}}+\frac{U}{L_{1}+\frac{2 K}{X_{1}}}=f_{3}
\end{aligned}
$$


Onde $\mathrm{X}_{1}$ é a posição da bola, $\mathrm{X}_{2}$ é a velocidade da bola, $\mathrm{X}_{3}$ é a corrente eletromagnética aplicada, a entrada de controle $U$ é a voltagem elétrica aplicada na bobina eletromagnética, $m$ é a massa da bola, $\mathrm{g}$ é a aceleração gravitacional, $\mathrm{K}$ é uma constante de força, $\mathrm{R}$ é a resistência da bobina, $\mathrm{L}_{1}$ é a indutância da bobina e $\mathrm{L}_{0}$ é a indutância da bobina sem a bola metálica. Os parâmetros são:

\begin{tabular}{|c|}
\hline Valores dos Parâmetros \\
\hline $\mathrm{m}=0.014 \mathrm{Kg}$ \\
\hline $\mathrm{g}=9.81 \mathrm{~m} / \mathrm{s}^{2}$ \\
\hline $\mathrm{K}=4.944 \times 10^{-6} \mathrm{Nm}^{2} / \mathrm{A}^{2}$ \\
\hline $\mathrm{R}=5.2 \Omega$ \\
\hline $\mathrm{L}_{1}=0.027 \mathrm{H}$ \\
\hline $\mathrm{L}_{0}=0.0011 \mathrm{H}$ \\
\hline
\end{tabular}

Tabela 1: Valores dos Parâmetros [6].

Este é um sistema dinâmico não-linear e não pode ser controlado técnicas de controle lineares tradicionais. Para controlar este tipo de sistema não-linear, técnicas avançadas são usadas, como $\mathrm{H} \infty$ [7], modos deslizantes [6] e estrutura variável [5]. Neste trabalho são comparadas três técnicas de controle não-lineares: PD (Proporcional-Derivativo) com sistema linearizado no ponto de equilíbrio, linearização por realimentação e SDRE (State-Dependent Riccati Equation).

\section{Técnicas de Controle}

Nesta seção são descritas as três técnicas de controle utilizadas.

A lei de controle PD (Proporcional-Derivativo) é dada por:

$$
U=K_{P}\left(X_{r e f}-X\right)-K_{D} \dot{X}
$$

onde $\mathrm{X}$ é a posição, $\mathrm{X}^{\prime}$ é a velocidade, $\mathrm{X}_{\text {ref }}$ é a referência para o controle e $\mathrm{K}_{\mathrm{P}}$ e $\mathrm{K}_{\mathrm{D}}$ são os ganhos do controle PD.

A técnica linearização por realimentação é a mais complexa de ser projetada e implementada. Esta técnica é muito usada na comunidade de controle. A ideia principal é transformar algebricamente um sistema dinâmico não-linear em um sistema dinâmico linear e aplicar uma técnica de controle linear ao sistema [4,8]. Há dois tipos de linearização, entradaestado e entrada-saída. Neste trabalho é usada a linearização entrada-estado. Considere o seguinte sistema não-linear:

$$
\dot{X}=f(X, U)
$$

O objetivo é projetar uma entrada de controle U. A linearização entrada-estado resolve este problema em dois passos. Primeiro, encontramos uma transformação de estado:

$$
Z=Z(X)
$$

e uma transformação de entrada:

$$
U=U(X, v)
$$

finalmente, transformamos o sistema não-linear em uma forma linear:

$$
\dot{Z}=A Z+b v
$$

Então usamos um controle linear tradicional para encontrar v. Na Figura 2, a técnica de controle pode ser vista graficamente. 


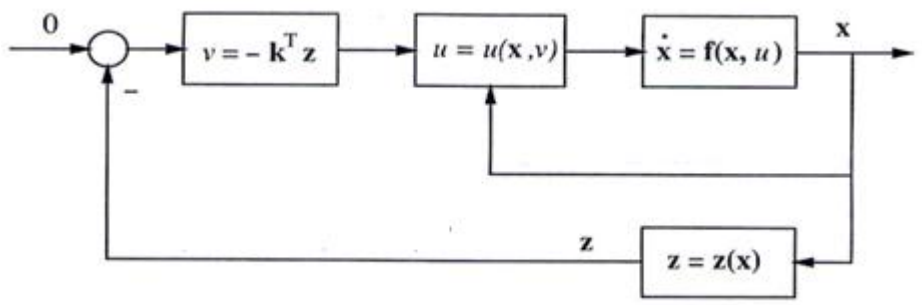

Figure 2: Linearização por realimentação [8].

O método de controle SDRE (State-Dependent Riccati Equation) tem recebido muita atenção da comunidade de controle atualmente [1]. Esta estratégia é muito eficiente para controle de realimentação não-linear. $O$ método representa o sistema não-linear em uma estrutura linear que tem matrizes dependentes de estado e minimiza um índice de performance quadrático. $\mathrm{O}$ algoritmo resolve, para cada ponto no espaço de estados, a equação algébrica de Riccati e dependente do estado. Por causa disto este método chama-se equação de Riccati dependente do estado (State-Dependent Riccati Equation).

Dado o sistema não-linear:

$$
\dot{X}=f(X)+g(X) U
$$

O sistema precisa ser transformado na seguinte forma:

$$
\dot{X}=A(X) X+B(X) U
$$

A lei de controle de realimentação que minimiza o índice de performance quadrático:

$$
J=\int_{0}^{\infty}\left[X(t)^{T} Q(X) X(t)+U(t)^{T} R(X) U(t)\right] d t
$$

é:

$$
U=-R^{-1}(X) B^{T}(X) P(X) X
$$

A matriz $\mathrm{P}(\mathrm{X})$ pode ser obtida pela equação de Riccati:

$$
\mathrm{P}(\mathrm{x}) \mathrm{A}(\mathrm{x})+\mathrm{A}^{\mathrm{T}}(\mathrm{x}) \mathrm{P}(\mathrm{x})+\mathrm{Q}(\mathrm{x})-\mathrm{P}(\mathrm{x}) \mathrm{B}(\mathrm{x}) \mathrm{R}^{-1}(\mathrm{x}) \mathrm{B}^{\mathrm{T}}(\mathrm{x}) \mathrm{P}(\mathrm{x})=0
$$

$\mathrm{Q}(\mathrm{X})$ e $\mathrm{R}(\mathrm{X})$ são parâmetros de projeto e são positivos definidos.

\section{Resultados Simulados}

Nesta seção são mostradas as performances das três técnicas de controle. O objetivo é manter a bola metálica na posição $0.012 \mathrm{~m}$. A posição inicial é $0.009 \mathrm{~m}$. Os resultados simulados com o software Matlab são mostrados abaixo. O método numérico para resolver o sistema não-linear é o Runge-Kutta de quarta ordem [2].

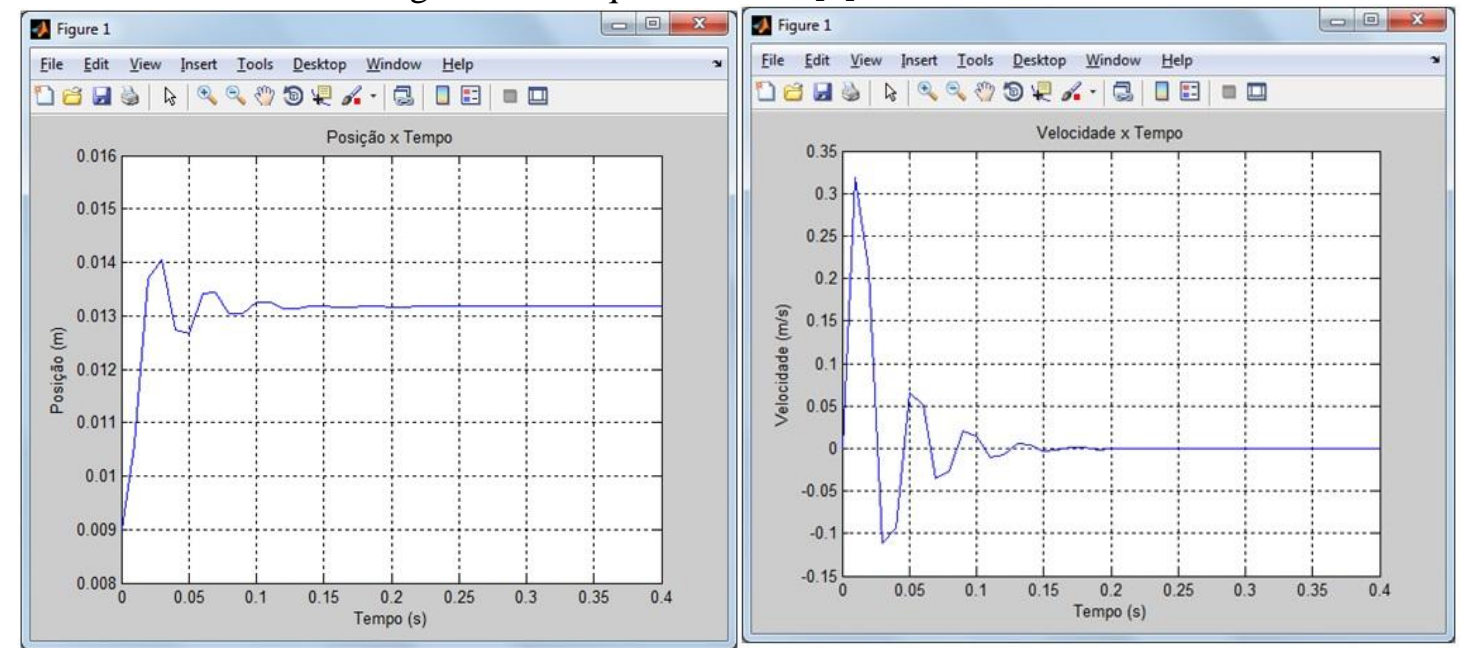




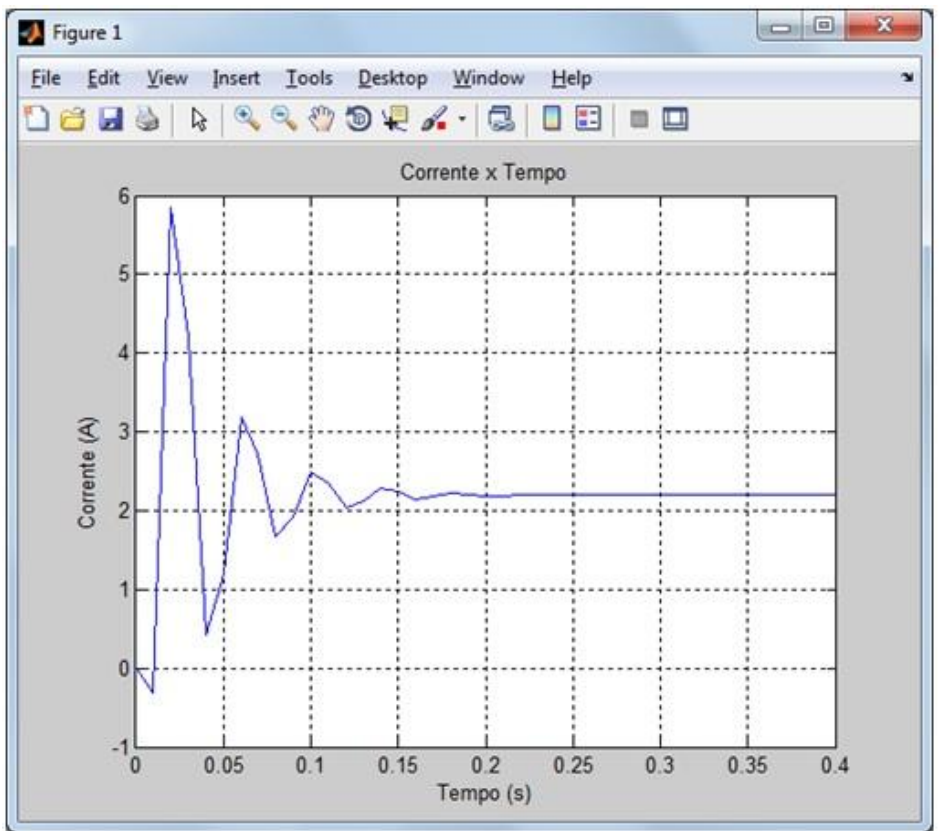

Figure 3: Posição, velocidade e corrente para a técnica de controle PD.

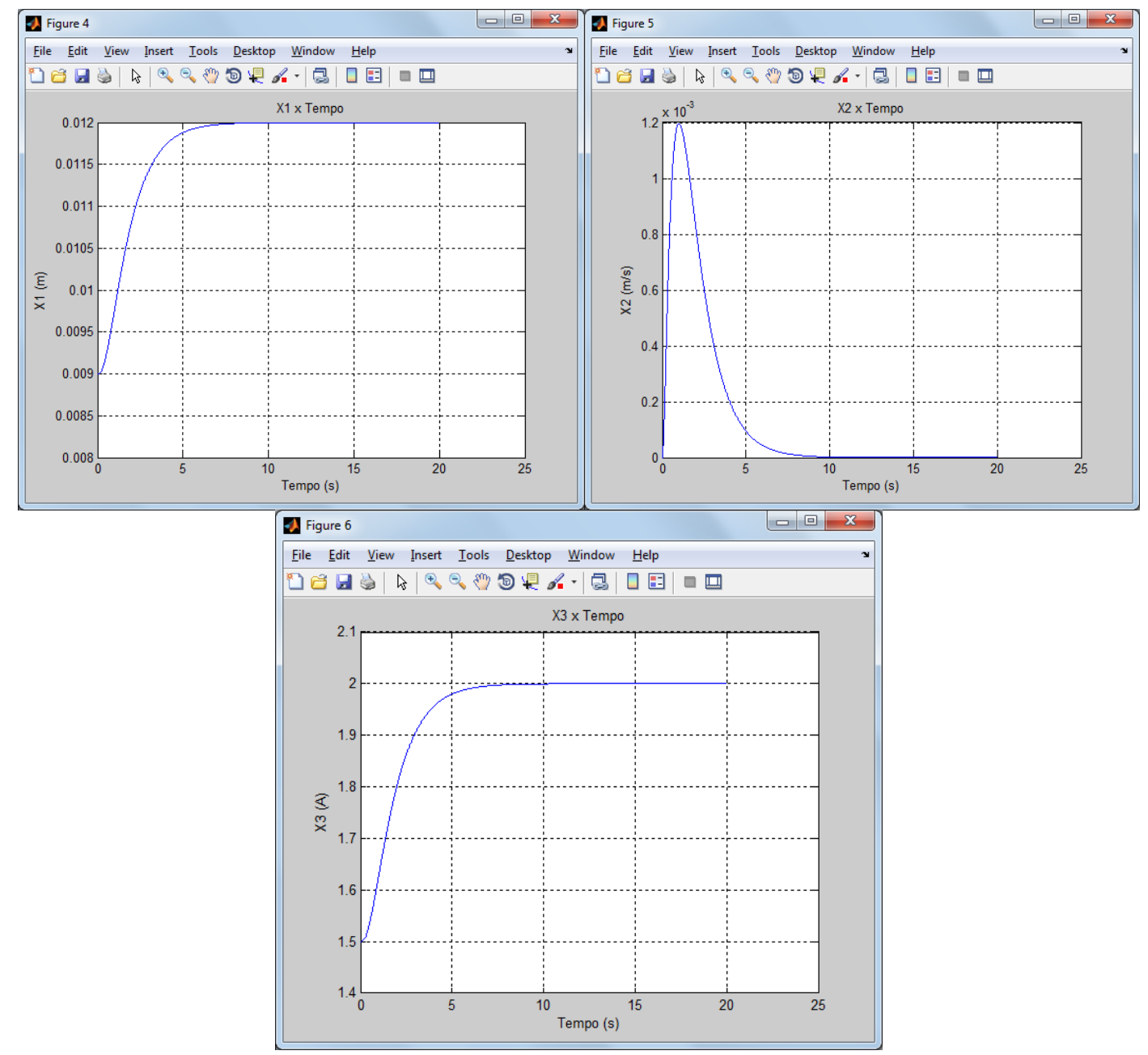

Figure 4: Posição, velocidade e corrente para a técnica de controle linearização por realimentação. 


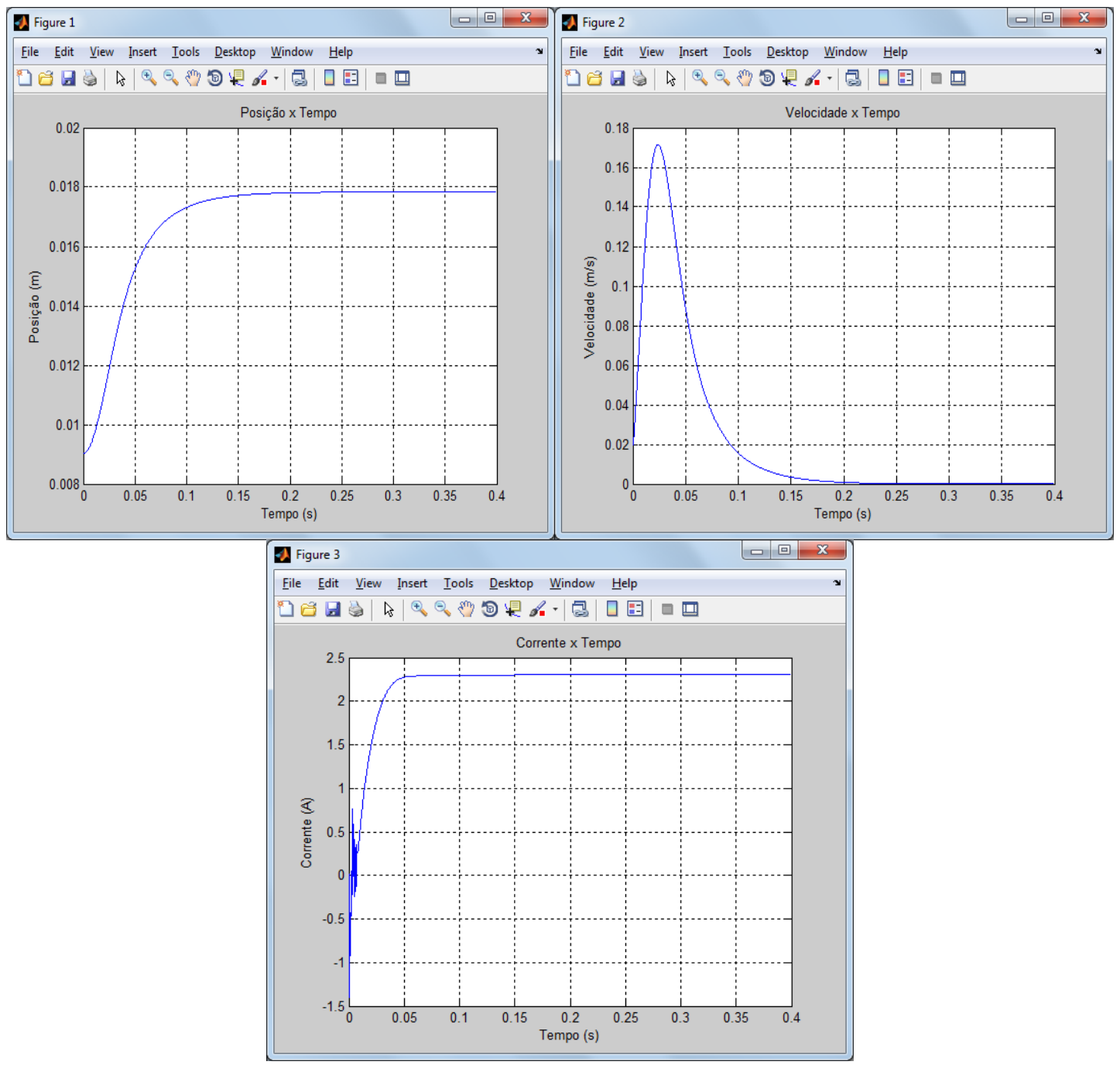

Figure 5: Posição, velocidade e corrente para a técnica de controle SDRE.

Analisando os resultados das simulações pode-se ver que a técnica de controle linearização por realimentação apresentou a melhor performance. A pior performance pode ser vista na técnica de controle SDRE. Isto ocorre devido a grande quantidade de cálculos e a performance de controle depende da escolha de A, B, Q e R em (8) e (9). Há a possibilidade várias escolhas para estas matrizes. A técnica de controle PD apresentou uma boa performance, no entanto, não é uma solução global e só vale próximo ao ponto de equilíbrio.

\section{Conclusões e Trabalhos Futuros}

Neste trabalho nós apresentamos o estudo e a implementação de três técnicas de controle aplicadas ao sistema de levitação magnética. As técnicas de controle são PD (Proporcional-Derivativo) com o sistema linearizado no ponto de equilíbrio, linearização por realimentação e SDRE (State-Dependent Riccati Equation). Analisando os resultados, a técnica linearização por realimentação teve a melhor performance e a técnica SDRE teve a pior performance.

Os principais trabalhos futuros são a aplicação de outros tipos de técnicas avançadas e não-lineares de controle como controle adaptativo, redes neurais e técnicas Fuzzy. 


\section{Referências}

[1] Çimen, State-Dependent Riccati Equation (SDRE) Control: a Survey, Proceedings of the 17th World Congress the International Federation of Automatic Control, 2008.

[2] Chapra, "Numeric Methods for Engineers", Mcgraw Hill, 2001.

[3] Kirk, "Optimal Control Theory: An Introduction", Princeton Hall Englewood Cliffs, 1970.

[4] Khalil, "Nonlinear Systems", Prentice Hall, 2002.

[5] Kharaajoo, High Performance Variable Structure Control of a magnetic Levitation System, Asian Journal of Control, Vol.4, No.3, 2002.

[6] Khemissi, Control Using Sliding Mode of the Magnetic Suspension System, International Journal of Electrical and Computer Sciences, Vol.10, No.3.

[7] Shen, $\mathrm{H} \infty$ Control and Sliding Mode Control of Magnetic Levitation System, Asian Journal of Control, Vol.4, No.3, 2002.

[8] Slotine, "Applied Nonlinear Control", Prentice Hall, 1991. 